\title{
ABORDAGEM AO PACIENTE INTOXICADO NO CONTEXTO DA TENTATIVA DE SUICÍDIO: UM RELATO DE EXPERIÊNCIA
}

\section{APPROACH TO THE INTOXICATED PATIENT IN THE CONTEXT OF SUICIDE'S ATTEMPT: AN EXPERIENCE REPORT}

Alba Rossana Vieira Costa (vieira.albarossana@gmail.com) ${ }^{1}$ Aline de Paula Rêgo Graciano Luz (aline_grac@yahoo.com.br) ${ }^{2}$ Emanuelle Silvino Coelho Martins Mestre (emanuellemestre@hotmail.com) ${ }^{3}$ Rafaela Simão de Abrantes (rafaelaabrantes19@gmail.com) ${ }^{4}$ Priscilla Kelly Policarpo Falcão (pri_policarpo@hotmail.com) ${ }^{5}$

RESUMO: OBJETIVO: O presente estudo relata a experiência graduandas em Enfermagem e como estagiárias/ plantonistas no Centro de Informações e Assistência Toxicológica de Campina Grande (CIATox-CG), com ênfase nos casos de intoxicação no contexto da tentativa de suicídio. METODOLOGIA: Trata-se de um estudo descritivo, do tipo relato de experiência, baseado na vivência de estágio no CIATox-CG que funciona no Hospital de Emergência e Trauma Dom Luiz Gonzaga Fernandes (HETDLGF), no período de setembro de 2016 a setembro de 2017. RESULTADOS: A atuação no serviço e as vivências de atendimentos em casos de intoxicações constituíram um diferencial na formação das estagiárias enquanto futuras enfermeiras. CONCLUSÃO: Tal experiência foi concluída de maneira positiva e avaliada como uma oportunidade ímpar de desenvolver habilidades profissionais, intelectuais e humanas.

Palavras chave: Intoxicação. Saúde Pública. Suicídio.

ABSTRACT: OBJECTIVE: The present study reports on the experience of undergraduate nursing students and interns / on-duty staff at the Campina Grande Toxicological Information and Assistance Center (CIATox-CG), with emphasis on cases of intoxication in the context of attempted suicide. METHODOLOGY: This is a

\footnotetext{
${ }^{1}$ Residente em Enfermagem em UTI - COREMU/IMIP.

${ }^{2}$ Mestranda em Saúde Pública pela Universidade Estadual da Paraíba (UEPB).

${ }^{3}$ Residente em Enfermagem Cirúrgica- COREMU/IMIP.

${ }_{5}^{4}$ Mestranda em Saúde Pública pela Universidade Estadual da Paraíba (UEPB).

${ }^{5}$ Mestranda em Saúde Pública pela Universidade Estadual da Paraíba (UEPB).
} 
descriptive study, an experience report, based on the internship experience at CIATox-CG that works at Dom Luiz Gonzaga Fernandes Emergency and Trauma Hospital (HETDLGF), from September 2016 to September 2017. RESULTS: The performance in the service and the experiences of care in cases of intoxication constituted a differential in the training of interns as future nurses. CONCLUSION: This experience was concluded in a positive way and evaluated as a unique opportunity to develop professional, intellectual and human skills.

KEYWORDS: Intoxication. Public health. Suicide. 


\section{INTRODUÇÃO}

O suicídio tem figurado, no cenário mundial, como a segunda maior causa de morte entre jovens na faixa etária dos 15 aos 29 anos de idade. Os dados divulgados pelo Relatório Global sobre o Suicídio da Organização Mundial da Saúde são alarmantes e mostram um número cada vez maior desses casos, de acordo com o mesmo, no mundo aproximadamente 800 mil pessoas cometem suicídio por ano, o que representa uma morte a cada 40 segundos (OMS, 2014).

A palavra suicídio origina-se do latim "sui" de si, a si próprio e "caedere" de bater, golpear, matar (ROEHE; DUTRA, 2017). A procedência dessa palavra indica o ato de violência infligido a si mesmo. O sociólogo francês Émile Durkhein em sua obra "O suicídio", define esse ato como sendo todo caso de morte resultante da ação da própria vítima, desde que ela saiba que produziria esse resultado, enquanto que a tentativa de suicídio seria o ato já produzido, no entanto interrompido antes que este pudesse provocar a morte (DURKHEIN, 2000).

A ingestão de praguicidas, o enforcamento e o uso de armas de fogo encontram-se entre os meios mais frequentemente utilizados para o suicídio a nível mundial (OMS, 2014). Sendo assim, observa-se que a intoxicação encontra-se entre as três principais formas de suicídio. Define-se intoxicação como sendo um processo patológico causado por substâncias químicas endógenas ou exógenas e caracterizado por desequilíbrio fisiológico, em consequência das alterações bioquímicas no organismo (OGA; CAMARGO; BATISTUZZO, 2014).

No Brasil, em 2013 o Sistema Nacional de Informações TóxicoFarmacológicas (SINITOX) registrou cerca de 7 mil casos de tentativas de suicídio por intoxicação em todo o país (SINITOX, 2013). O Centro de Informações e Assistência Toxicológica do município de Campina Grande- PB (CIATox-CG), registrou no período de janeiro de 2011 a dezembro de 2015, 3.019 casos de intoxicação, dentre esses, 1.038 estavam relacionados à tentativa de suicídio (COSTA et al., 2017). 
Diante de dados tão alarmantes, esses casos têm se mostrado um problema de saúde pública e caracterizam emergências cada vez mais frequentes nos serviços de saúde (VIDAL; CONTIJO; LIMA, 2013). Apesar de ser um tema envolto em tabus e preconceitos, cada vez mais se tem notado a necessidade de abordar essa temática. Por fazerem parte de um grupo de estagiárias do CIATox-CG, e serem discentes do curso de Enfermagem, diante dos vários casos surgidos durante o período do estágio, e pela inquietação pelo elevado número de casos atendidos no serviço, surgiu o interesse na abordagem desse, que tem como objetivo relatar as atividades desenvolvidas em um serviço de referência na assistência à vítimas de tentativa de suicídio, além de expressar as dificuldades e barreiras enfrentadas no atendimento ao cliente que se intoxica com a finalidade de autoextermínio.

\section{METODOLOGIA}

Trata-se de um estudo descritivo, do tipo relato de experiência. As pesquisas descritivas têm como caráter primordial a descrição das características de determinada população ou fenômeno, para isso utilizam-se de técnicas tais como o questionário e a observação sistemática na coleta de dados (GIL, 2002). O relato de experiência é uma ferramenta da pesquisa descritiva que reflete uma ação ou conjunto de ações que abordam situações vivenciadas no âmbito profissional que interessem ao meio científico (CAVALCANTE; LIMA, 2012).

O presente estudo é fundamentado na vivência das autoras durante o desenvolvimento de suas atividades no Programa de Extensão em Toxicovigilância e Atenção Toxicológica, do departamento de Farmácia da Universidade Estadual da Paraíba (UEPB). Para a opercionalização desse estudo, foi utilizada a observação em campo, bem como o diário de campo.

As referidas atividades foram desenvolvidas no período de setembro de 2016 a setembro de 2017, onde as autoras atuaram como plantonistas do Centro de Informações e Assistência Toxicológica - CIATox-CG, situado no Hospital de Emergência e Trauma Dom Luiz Gonzaga Fernandes, na cidade de Campina 
Grande-PB. O HETDLGF é o hospital referência em trauma para 203 municípios da Paraíba, além de atender os municípios do Rio Grande do Norte, Pernambuco e Ceará. O serviço tem capacidade para 292 leitos, e disponibiliza uma equipe médica de 340 médicos, sendo 64 em regime de plantão presencial 24 horas, além disso dispõe de 6 salas operatórias no bloco cirúrgico. Em balanço recente, o HETDLGF divulgou que apenas no primeiro trimestre de 2017 atendeu 18.568 pessoas e realizou 1.860 cirurgias (GOVERNO DA PARAÍBA, 2017).

\section{REVISÃO DE LITERATURA}

Desde os tempos mais remotos o homem buscava entender os efeitos produzidos por substâncias oriundas de plantas e animais. Relatos de envenenamento estão presentes desde a antiguidade, onde por vezes o poder exterminador dos venenos era utilizado como arma contra inimigos, sendo assim pode-se dizer que a história da Toxicologia acompanha a própria história da civilização (OGA; CAMARGO; BATISTUZZO, 2014).

A intoxicação é definida como o efeito prejudicial causado por exposição a uma substância química (FILHO; CAMPOLINA; DIAS, 2013). Enquanto que a Toxicologia é a ciência que estuda os efeitos nocivos decorrentes das interações de tais substâncias com o organismo. Os primeiros relatos sobre intoxicação foram identificados no Papiro de Ebers (1500 a.C.), de origem egípcia que listava cerca de 800 ingredientes ativos. Nele havia relatos de intoxicação por chumbo, cobre, plantas venenosas entre outros (OGA; CAMARGO; BATISTUZZO, 2014; FILHO; CAMPOLINA; DIAS, 2013).

Atualmente, com o avanço da ciência na área da saúde e a descoberta de novos tratamentos, surgiu também uma grande quantidade de novas fórmulas e medicamentos (PINTO et al., 2014). O aumento da quantidade de medicamentos e produtos químicos nos últimos anos tem aumentado significativamente o risco de intoxicações. A aquisição de medicamentos se tornou mais fácil, o que intensifica a exposição da população a esses agentes. Diversos autores corroboram com a ideia 
de que grande parte das intoxicações e reações adversas graves são causadas por esses fármacos (COSTA et al., 2017; FILHO;

CAMPOLINA; DIAS, 2013; MOTA et al., 2012; TELES et al., 2013; VIEIRA; SANTANA; SUCHARA, 2015).

A intoxicação é apontada atualmente como um dos principais métodos utilizados por pessoas no intento do suicídio (OMS, 2014). O suicídio constitui um grave problema de saúde pública e representa um desafio para a assistência de saúde. Muitos são os fatores que podem predispor uma pessoa em suas ideações suicidas e leva-la ao suicídio. Durkheim (2000) trata o suicídio como um fenômeno social, denominando-o de patologia social encontrada já nas primeiras etapas da evolução e até mesmo com certa virulência em algumas sociedades na antiguidade.

Entre os fatores microssociais que podem induzir uma pessoa a atentar contra sua própria vida estão perdas pessoais, relacionamentos rompidos, isolamento social, violências, problemas legais e no trabalho. Como fatores ambientais destacam-se estressores de vida, facilidade de acesso aos meios que possibilitem o autoextermínio e exposição por efeito da vizinhança ou meios de comunicação (BAHIA et al., 2017).

Os medicamentos junto aos agrotóxicos, principalmente o "chumbinho", figuram entre os agentes mais presentes nas tentativas de suicídio por intoxicação (COSTA et al., 2017; DANTAS et al., 2013; SANTOS; LEGAY; LOVISI, 2013; VIEIRA; SANTANA;

SUCHARA, 2015). Sendo assim, uma abordagem inicial assertiva ao paciente intoxicado fará toda a diferença no desfecho do quadro. Como em toda emergência, a conduta terapêutica requer uma avaliação rápida da clínica apresentada pelo intoxicado e a tomada de medidas que visem a estabilização e manutenção das condições vitais do paciente (TAVARES; OLIVEIRA, 2012).

A identificação do agente utilizado na tentativa de suicídio é de suma importância para a tomada de decisões no tratamento da intoxicação, no entanto nem sempre é possível determinar o agente tóxico. Nesses casos, a observação dos sinais e sintomas manifestados e o reconhecimento precoce da síndrome neurotóxica envolvida irão determinar a solicitação de testes diagnósticos e o tratamento específico. Entende-se por síndrome neurotóxica o conjunto de sinais e 
sintomas produzidos por substâncias químicas, que apesar de diferentes, produzem efeitos semelhantes (NÓBREGA et al., 2015).

A sistematização do atendimento ao paciente intoxicado deve incluir a avaliação e atendimento primário, avaliação clínica e laboratorial, aplicação das medidas de descontaminação e/ou tratamento específico, bem como o tratamento sintomático e de suporte (TAVARES; OLIVEIRA, 2012). A abordagem inicial é decisiva no prognóstico do paciente, devendo ser implementado o suporte avançado de vida possibilitando uma boa ventilação e respiração, além de um monitoramento efetivoda circulação a fim de identificar irregularidades no ritmo cardíaco (FILHO; CAMPOLINA; DIAS, 2013).

Concomitante ao suporte avançado de vida, os membros da equipe devem coletar o máximo de informações possíveis sobre o paciente e as condições em que ocorreram a intoxicação junto aos familiares e os socorristas que ofereceram o atendimento primário. Se o paciente estiver em condições de falar esses dados devem ser coletados com ele e depois confirmados com os acompanhantes. Informações acerca da substância envolvida, doenças prévias e passado de tentativas de suicídio são muito úteis e podem nortear o tratamento específico (FILHO; CAMPOLINA; DIAS, 2013).

Deve ser dada atenção especial ao exame físico, avaliando padrão respiratório, nível de consciência, funções vitais como um todo e exame neurológico no paciente comatoso. A presença de odores característicos, lesões na pele e mucosas além de fasciculações musculares e alterações na temperatura corporal são achados que podem indicar o agente tóxico envolvido (FILHO; CAMPOLINA; DIAS, 2013).

No intuito de diminuir a exposição do organismo ao agente ou até mesmo interromper sua absorção são tomadas medidas de descontaminação específicas para cada via de administração. Sendo a via oral a mais comum nas intoxicações, serão aqui abordadas as medidas de descontaminação que a ela correspondem (DANTAS et al., 2013; SANTOS; NETO; CUNHA, 2015; TAVARES; OLIVEIRA, 2012).

A eficácia da descontaminação do trato gastrointestinal por meio da lavagem gástrica ou por êmese induzida é controversa. A indução da êmese e o uso do 
xarope de ipeca para este fim, já não são mais indicados, dentre as contraindicações dessa prática estão a possibilidade dos vômitos promoverem a passagem do material tóxico para o intestino delgado e assim aumentar a absorção. Quanto a lavagem gástrica, apesar de muito vista nos serviços de emergência, sua utilização deveria se dar ocasionalmente para remover fármacos e venenos ingeridos em quantidade maciça. Existe uma maior probabilidade de eficácia da lavagem gástrica se feita nos primeiros 30-60 minutos, no entanto ainda será útil mais tardiamente nos casos de agentes que retardam o esvaziamento gástrico (OLSON et al., 2014).

O carvão ativado é um elemento particularmente importante e muito útil na maioria das intoxicações por via digestiva. Trata-se de um pó altamente adsorvente, feito a partir da destilação de polpa da madeira e devido sua grande área superficial é eficaz na adsorção da maioria das toxinas quando administrado na proporção certa. Em contrapartida, o carvão não é capaz de adsorver substâncias como metais pesados, lítio, fluoreto, ferro e cianeto (OLSON et al., 2014).

Medicamentos que atuam como antagonistas podem ser utilizados em algumas intoxicações específicas, são exemplos desses fármacos o flumazenil e a naloxona que se opõem a ação dos benzodiazepínicos e dos opióides respectivamente. Podemos destacar também o papel da atropina que nas intoxicações por carbamatos e organofosforados, durante o desenvolvimento de uma síndrome colinérgica antagoniza os efeitos colinérgicos nos receptores muscarínicos (ALMEIDA; COUTO; CHEQUER, 2016).

Em pacientes que já não se beneficiam com as medidas de descontaminação citadas, pode-se lançar mão de outros métodos que visam a excreção do toxicante como por exemplo, a diurese forçada e a manipulação do $\mathrm{pH}$ urinário, ou ainda métodos extracorpóreos como a hemodiálise, hemoperfusão e exsanguineotransfusão. Todos esses métodos possuem contraindicações e por isso deve ser considerado o risco-benefício na escolha de um deles como tratamento (FILHO; CAMPOLINA; DIAS, 2013; OLSON et al., 2014).

Diante da complexidade envolvida no atendimento aos casos de intoxicação atrelados à tentativa de suicídio, se torna imprescindível a ação da equipe interdisciplinar, na comunhão de esforços que visem o atendimento ao paciente enquanto um ser biopsicossocial. A atuação do enfermeiro inserido nessa equipe 
repercute favoravelmente para a redução dos índices de recidivas destas intoxicações (SANTOS; NETO; CUNHA, 2015). A capacitação de todos os membros da equipe multiprofissional se torna indispensável tendo em vista que o prognóstico do paciente depende da identificação e manejo adequado da intoxicação.

\section{RESULTADOS E DISCUSSÕES}

Em 2005, a Agência Nacional de Vigilância Sanitária - ANVISA publicou a RDC 19/2005, através da qual cria a Rede Nacional de Centros de Informação e Assistência Toxicológica - RENACIAT, além de aprovar diretrizes para a qualificação dos Centros de Informação e Assistência Toxicológica - CIATs (ANVISA, 2005). Estes são centros especializados em fornecer informações e orientações acerca de intoxicações e envenenamentos, assim como sobre a toxicidade de substâncias químicas e biológicas e os riscos que elas oferecem a saúde (COSTA et al., 2017).

Anualmente, o departamento de Farmácia da Universidade Estadual da Paraíba - UEPB, realiza o processo seletivo para o provimento de vagas para estagiários/ plantonistas do Centro de Informação e Assistência Toxicológica CIATox-CG. Participam do processo alunos dos cursos de Enfermagem, Farmácia, Medicina e Biologia oriundos de Instituições de Ensino Públicas e Privadas de Campina Grande.

A seleção é composta por prova objetiva que aborda temas relativos à Toxicologia e a Toxinologia. Uma vez aprovado nessa fase, o então estagiário passa por treinamento em serviço que acontece no Hospital de Emergência e Trauma Dom Luís Gonzaga Fernandes - HETDLGF. Esse treinamento é conduzido pelo plantonista da cota/ano anterior e tem duração de aproximadamente um mês. Após o treinamento o novo plantonista estará apto para assumir seus próprios plantões e assim desenvolver as atividades propostas pelo programa.

O plantonista deve cumprir a carga horária semanal de 12 horas, podendo estas serem divididas em dois turnos, ou de modo integral durante o dia ou a noite, 
havendo a possibilidade do desempenho de suas atividades aos finais de semana e feriados, tendo em vista que o serviço funciona ininterruptamente.

Durante sua atuação no Centro de Informação e Assistência Toxicológica CIATox- CG, o plantonista desenvolve atividades junto aos pacientes e equipe multiprofissional do serviço, além de atender a comunidade em geral. Podemos elencar as atividades em:

1. Atendimentos telefônicos aos que buscam informações através do Disque Intoxicação (0800- 722- 6001). O público que busca informações através deste serviço é diversificado e composto pela população em geral mas, também por profissionais que se encontram em outros serviços de saúde e buscam esclarecimentos acerca de intoxicações e acidentes por animais peçonhentos.

2. Notificação de casos de intoxicação e acidentes por animais peçonhentos utilizando as fichas especificas para estes fins. Os casos de intoxicação são de notificação compulsória e alimentam o SINAN - Sistema de Informação de Agravos de Notificação.

3. Serviço de consultoria junto a equipe multiprofissional do HETDLGF, fornecendo informações sobre diretrizes e manejos clínicos sempre que solicitados.

4. Acompanhamento, avaliação e evolução clínica dos pacientes intoxicados ou acidentados por animais peçonhentos durante toda a permanência destes no serviço, sendo feita evolução em ficha de evolução respeitando os princípios da semiologia no relato do quadro do paciente.

5. Avaliação periódica dos exames laboratoriais dos pacientes internos e o preenchimento de formulário específico para acompanhamento desses resultados.

6. Preenchimento do Livro de Admissões e Altas do serviço.

7. Informatização das fichas notificadas pelo plantonista em planilha do Excel.

8. Participação em reuniões, seminários e palestras propostas pela coordenação do serviço.

9. Troca diária de saberes entre os membros do CIATox-CG e os profissionais do HETDLGF na prestação da assistência. Além da educação promovida pelo plantonista junto ao paciente, afim de evitar novos acidentes e agravos.

10. Desenvolvimento de pesquisas utilizando dados epidemiológicos do serviço. 
O CIATox-CG proporciona ao estagiário uma gama de novas vivências que muitas vezes não puderam ser oportunizadas durante os estágios curriculares da graduação. Torna-se possível uma aproximação mais efetiva da enfermagem, sendo possível observar sua atuação nos mais diversos setores de um hospital de grande porte, seja nas alas de internação, nas UTI's, na emergência ou na pediatria.

Foi possível o reconhecimento da estrutura e funcionamento de um serviço referência em urgência, emergência e traumatologia, nos mais diversos níveis de complexidade. A classificação de risco vista durante a graduação se tornou mais nítida após a vivência da rotina de um serviço de média e alta complexidade.

Foram desenvolvidas habilidades como exame físico, histórico de enfermagem, evolução de enfermagem, avaliação de exames laboratoriais. Adquiriuse maior intimidade com grupos farmacológicos e sua atuação no organismo humano, bem como maior traquejo ao lidar com acidentes por animais peçonhentos, atendimentos de urgência e emergência e no próprio diálogo com a equipe interdisciplinar com vistas ao cuidado integrado do paciente.

A experiência de serem inseridas na equipe interdisciplinar trouxe grandes aprendizados e também grandes desafios. Assim como é próprio das relações interpessoais, com cada profissional conseguiu-se estabelecer uma relação diferente, alguns profissionais se mostraram abertos ao diálogo e discussão acerca de condutas, avaliação do paciente e interpretação de exames, enquanto que outros não se mostraram igualmente receptivos, sendo por vezes resistentes e até mesmo incrédulos quanto às orientações. Na maioria das vezes essas barreiras foram sendo vencidas ao custo de muito estudo e contribuição efetiva nos casos.

Outro desafio enfrentado foi o atendimento ao paciente intoxicado como tentativa de suicídio. Esse tipo de ocorrência coloca o plantonista diante de conflitos éticos e morais, onde é necessário que o mesmo se dispa de todos os preconceitos e bagagem pessoal para acolher o paciente, escutá-lo e tentar ajudá-lo da melhor maneira possível, ao mesmo tempo mantendo uma distância necessária. O ponto positivo é que no serviço em questão a equipe interdisciplinar conta com psicólogos e assistentes sociais que são de grande valia nesses casos. Como ponto negativo podemos ressaltar que nem todos os membros da equipe conseguem desenvolver empatia por esses pacientes muitas vezes os tratando de forma áspera e fria, 
julgando que os mesmos estão ocupando o leito de alguém que está "realmente doente". Todavia, as experiências positivas se sobrepuseram às negativas. O CIATox-CG é um serviço respeitado e tem sua importância reconhecida pelo HETDLGF e consegue rotineiramente fazer a diferença na assistência prestada ao paciente intoxicado. Além de ser um excelente meio formador de profissionais das mais diversas áreas das ciências da saúde.

\section{CONSIDERAÇÕES FINAIS}

O Centro de Informação e Assistência Toxicológica - CIATox-CG, constitui um serviço de grande relevância para o Hospital de Emergência e Trauma Dom Luís Gonzaga Fernandes - HETDLGF, serviço onde atua, bem como para todos os serviços de saúde que buscam orientações através de contato telefônico, contribuindo para o melhor atendimento e abordagem assertiva ao paciente intoxicado.

Enquanto unidade de ensino, pesquisa e extensão, forma profissionais de maneira ética e humana, capacitando futuros profissionais das mais diversas áreas da saúde no campo da Toxicologia. Tendo em vista a deficiência na maioria das grades curriculares dos cursos de graduação, no que diz respeito à esta área de estudo, o CIATox-CG constituí uma oportunidade única de aprofundamento numa área diferenciada.

A atuação junto a equipe interdisciplinar e as vivências de atendimentos em casos de intoxicações e acidentes por animais peçonhentos constituíram um diferencial na formação das estagiárias enquanto futuras enfermeiras. Tal experiência foi concluída de maneira positiva e avaliada como uma oportunidade ímpar de desenvolver habilidades profissionais, intelectuais e humanas na prestação de uma assistência holística ao paciente como ser biopsicossocial. 


\section{REFERÊNCIAS BIBLIOGRÁFICAS}

AGÊNCIA NACIONAL DE VIGILÂNCIA SANITÁRIA - ANVISA (2005). RDC $\mathbf{n}^{\circ} \mathbf{1 9}$, de de fevereiro de 2005. Disponível em < http://www.saude.mg.gov.br/images/ documentos/ RDC_19.pdf> Acessado em: 14/09/2017.

ALMEIDA, T. C. A; COUTO, C.C; CHEQUER, F.M.D. Perfil das intoxicações agudas ocorridas em uma cidade do centro-oeste de Minas Gerais. Eletronic Journal of Pharmacy, v. 13, n.3, p. 151-164. 2016. Disponível em: <http://revistas.ufg.br/index.php/REF/index>. Acesso em: 06 nov. 2017.

BAHIA, C.A. et al. Lesão autoprovocada em todos os ciclos da vida: perfil das vítimas em serviços de urgência e emergência de capitais do Brasil. Cienc. Saúde Coletiva, v.22, n.9, p. 2841-2850. 2017. Disponível em: <http://www.scielo.br/pdf/csc/v22n9/1413-8123-csc-22-092841.pdf>. Acesso em: 06 nov. 2017.

CAVALCANTE, B.L.L; LIMA, U.T.S. Relato de experiência de uma estudante de enfermagem em um consultório especializado em tratamento de feridas. J. Nurs Health, Pelotas, v.1, n.2, p. 94-103, jan-jun, 2012. Disponível em: <https://periodicos.ufpel. edu.br/ojs2/index.php/ enfermagem/article/view/3447/2832>. Acesso em: 01 set. 2017.

COSTA, A.R.V. et al. Tentativa de suicídio por intoxicação: Retrato de um problema mundial. In: CONGRESSO BRASILEIRO DE CIÊNCIAS DA SAÚDE, 2.,2017, Campina Grande, Anais eletrônicos...Campina Grande: Realize, 2017. Disponível em: <http://editorarealize.com.br/revistas/conbracis/anais.php>. Acesso em: 01 set. 2017.

DANTAS, J.S.S. et al. Perfil do paciente com intoxicação exógena por "chumbinho" na abordagem inicial em serviço de emergência. Rev. Elet. Enf, Goiania, v.15, n.1, p.54-60, janmar, 2013. Disponível em: < https://revistas.ufg.br/fen/article/view/15506>. Acesso em: 06 nov. 2017.

DURKHEIN, E. O suicídio: estudo de sociologia. São Paulo: Martins Fontes, 2000.

FILHO, A.A.; CAMPOLINA, D.; DIAS, M.D. Toxicologia na prática clínica. $2^{a}$ ed. Belo Horizonte: Folium, 2013. p. 1-25.

GIL, A.C. Como elaborar projetos de pesquisa. $4^{a}$ ed. São Paulo: Atlas, 2002, p. 42.

GOVERNO DA PARAÍBA. Hospital de Trauma de Campina Grande divulga balanço de atendimento do primeiro trimestre. Disponível em: <http://paraiba. pb.gov.br/hospital-detrauma-de-campina-grande-divulga-balanco-de atendimentos-do-primeiro-trimestre/>. Acesso em: 14 set. 2017.

MOTA, D.M et al. Perfil da mortalidade por intoxicação com medicamentos no Brasil,19962005: retrato de uma década. Rev. Ciênc e Saúde Coletiva, Rio de Janeiro, v.17, n. 1, p. 6170. 2012. Disponivel em: <http://www.scielo.br/scielo.php?script=sci_arttext\&pid=S1413$81232012000100009>$. Acesso em: 05 nov. 2017.

NÓBREGA, H.O.S et al. Intoxicações por medicamentos: uma revisão sistemática com abordagem nas síndromes tóxicas. Rev. Saúde e Ciência, Campina Grande, v.4, n.2, p.109119. 2015. Acesso em: 04 nov. 2017.

ORGANIZAÇÃO MUNDIAL DE SAÚDE - OMS (2014). Prevención del suicídio: um 
imperativo global. Washington, DC, 2014. Disponível em:<http://apps.who.int/iris/ bitstream/10665/136083/1/9789275318508_spa.pdf?ua=1\&ua=1>. Acesso em: 26 abr. 2017.

OGA, S.; CAMARGO, M.M.A.; BATISTUZZO, J.A.O. Fundamentos de Toxicologia. $4^{a}$ ed. São Paulo: Atheneu; 2014.

OLSON, K.R. et al. Manual de toxicologia clínica. $6^{\text {a }}$ ed. Porto Alegre: AMGH; 2014, p. 1- 56.

PINTO, G.M.F. et al. Estudo de descarte residencial de medicamentos vencidos na região de Paulinia (SP), Brasil. Rev. Eng Sanit Ambient, Rio de Janeiro, v. 19, n. 3. p. 219-224, jul- set, 2014. Disponível em: <http://www.scielo.br/scielo.php?script=sci_arttext\&pid=S141341522014000300219>. Acesso em: 02 nov. 2017.

ROEHE, M.V; DUTRA, E. Compreendendo narrativas sobre suicídio com base na analítica existencial de Martin Heidegger. Rev da Abordagem Gestáltica, Goiânia, v.23, n.1, p. 32- 41, jan-abri, $2017 . \quad$ Disponível em: <http://pepsic.bvsalud.org/scielo.php?script=sci_arttext\&pid=S1809-68672017000100005>.

Acesso em: 26 abr. 2017.

SANTOS, R.R; NETO, O.P.A; CUNHA, C.M. Perfil de vítimas de intoxicações agudas e assistência de enfermagem. Rev. Enferm Atenção Saúde, v.4, n.2, p.45-55, ago-dez, 2015. Disponivel em: <http://seer.uftm.edu.br/revistaeletronica/index.php/enfer/article/view/978/pdf>. Acesso em: 05 nov. 2017.

SANTOS, S.A; LEGAY, L. F; LOVISI, G.M. Substâncias tóxicas e tentativas e suicídios: considerações sobre acessos e medidas restritivas. Cad. Saúde Colet., Rio de Janeiro, v.21, n.1, p. 53-61. 2013. Disponível em: <http://www.scielo.br/pdf/cadsc/v21n1/a09.pdf>. Acesso em: 06 nov. 2017.

SISTEMA NACIONAL DE INFORMAÇÕES TÓXICO-FARMACOLÓGICAS - SINITOX(2013). Dados de intoxicação. Rio de Janeiro, 2013. Disponível em: <https://sinitox.icict. fiocruz.br/dados-nacionais>. Acesso em: 01 set. 2017.

TAVARES, E.O; OLIVEIRA, M.L.F. Padrões mínimos de atendimento inicial à urgência toxicológica para abordagem à criança intoxicada. Rev Rene, v.13, n.1, p. 147-157, 2012. Disponível em: <http://www.periodicos.ufc.br/rene/article/view/3785/2991>. Acesso em: 06 nov. 2017.

TELES et al. Papel dos medicamentos nas intoxicações causadas por agentes químicos em município da Bahia, no período de 2007 a 2010. Rev Ciênc Farm Básica Apl., v. 34, n. 2, p. 281-288, $2013 . \quad$ Disponível em: $<$ http://servbib.fcfar.unesp.br/seer/index.php/Cien_Farm/article/viewArticle/2407>. Acesso em: 05 nov. 2017.

VIDAL, C.E.L.; GONTIJO, E.C.D.M.; LIMA, L.A. Tentativas de suicídio: fatores prognósticos e estimativas do excesso de mortalidade. Cad. Saúde Pública, Rio de Janeiro. v.29, n.1, p. 175187, jan, 2013. Disponível em: <http://www.scielo.br/pdf/csp/v29n1/20. pdf>. Acesso em: 06 nov. 2017.

VIEIRA, L. P.; SANTANA, V. T. P.; SUCHARA, E. A. Caracterização de tentativas de suicídios por substâncias exógenas. Cad. Saúde Colet., Rio de Janeiro, v. 23, n. 2, p. 118-123. 2015. Disponível em: <http://www.scielo.br/pdf/cadsc/v23n2/1414-462X-cadsc-23-2- 118.pdf>. Acesso em: 24 abr. 2017. 